\title{
Functionalisation of Multi-Layer Graphene-Based Gas Sensor by Au Nanoparticles +
}

\author{
Laura Morelli ${ }^{1}$, Filiberto Ricciardella ${ }^{1}$, Max Koole ${ }^{2}$, Stefan Persijn ${ }^{3}$ and Sten Vollebregt ${ }^{1, *}$ \\ 1 Department of Microelectronics, Delft University of Technology, 2628 CD Delft, The Netherlands; \\ lmorelli1009@gmail.com (L.M.); filiberto.ricciardella@gmail.com (F.R.) \\ 2 VSParticle B.V., 2629 JD Delft, The Netherlands; info@vsparticle.com \\ 3 VSL-Dutch Metrology Institute, 2629 JA Delft, The Netherlands; SPersijn@vsl.nl \\ * Correspondence: s.vollebregt@tudelft.nl \\ + Presented at the 4th International Conference nanoFIS 2020-Functional Integrated nano Systems, \\ Graz, Austria, 2-4 November 2020.
}

Published: 7 December 2020

\begin{abstract}
A novel gas sensor based on multi-layered graphene (MLG) functionalised with gold nanoparticles (Au-NPs) is presented. We demonstrate for the first time that: (1) the signal saturates during the analyte exposure, something which does not occur in the pristine material and in graphene-based gas sensors in general; (2) the sign of the device current response is inverted. MLG is grown by chemical vapour deposition on pre-patterned CMOS-compatible Mo catalyst. The sensor is fabricated directly on the growth substrate, without any transfer of MLG. The Au-NPs are later deposited from an aerosol on the sensor at a specific controlled location, mitigating any additional patterning steps. The functionalised sensor is tested with $1 \mathrm{ppm}$ (part-per-million) of $\mathrm{NO}_{2}$ at room temperature.
\end{abstract}

Keywords: gas sensors; graphene; functionalisation; nanoparticles

\section{Introduction}

Graphene retrieves much attention as a promising material for gas sensing applications, because of its potential ppb-level (part-per-billion) sensitivity at room temperature (RT) and low 1/f noise. Graphene-based gas sensors working at RT exhibit enhanced sensitivity towards many gas molecules, e.g., $\mathrm{NO}_{2}, \mathrm{NH}_{3}$, and $\mathrm{H}_{2} \mathrm{O}$, thanks to the ambipolar nature of this material [1].

However, these gas-sensors are still affected by low selectivity, poor recovery and absence of response saturation, hampering their application in commercial devices. Functionalising the graphene with molecules, dopants or nanoparticles has been proposed as a method to improve selectivity by changing the electrical properties of the graphene. For instance, Au-NPs deposited on single-layer graphene have been shown to change the electrical doping, with a dependence on the coverage of the NPs [2]. In this work, we experimentally show that Au-NPs can effectively reverse the gas sensing behaviour of multi-layered graphene (MLG). Moreover, we present for the first time a saturating MLG-based gas sensor, working at RT.

\section{Methods}

The chemi-resistive gas sensor is illustrated in Figure 1a. The sensing layer was grown using chemical vapour deposition (CVD) at around $1000^{\circ} \mathrm{C}$ on a pre-patterned Mo catalyst. The device was prepared on the same substrate where MLG was selectively grown in pre-determined locations, avoiding the need for graphene transfer [3]. The Au-NPs, used to functionalise the graphene, were selectively deposited by inertial impaction from a solvent-free aerosol stream of Au-NPs generated by spark ablation in a commercially available NP generator. The sensor with pristine and 
functionalised MLG was exposed to $1 \mathrm{ppm}$ of $\mathrm{NO}_{2}$ at RT in dry $\mathrm{N}_{2}$, while biased at $1 \mathrm{~V}$. Pristine material was previously found to be sensitive to $\mathrm{NO}_{2}$ [4].

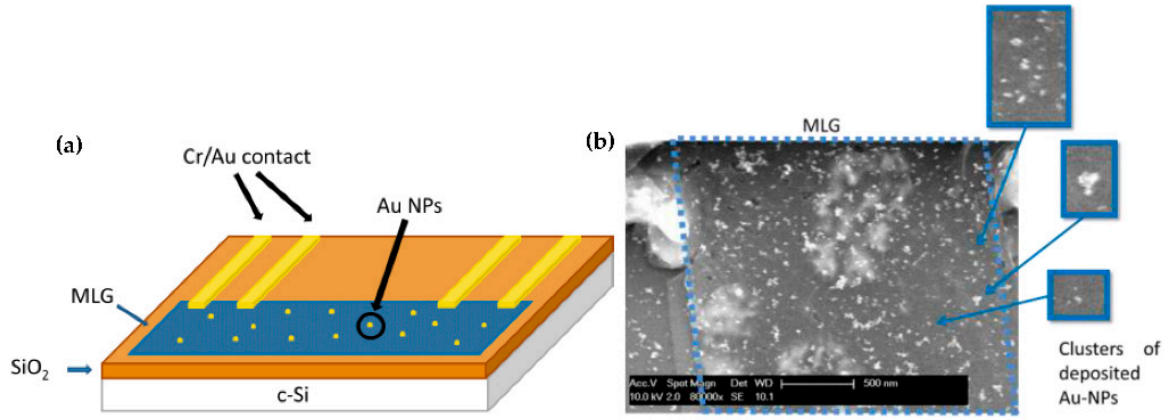

Figure 1. (a) Device schematic, on the multi-layered graphene (MLG) bar (206 $\mu \mathrm{m}$ long, $10 \mu \mathrm{m}$ wide) where the gold nanoparticles are deposited. The $\mathrm{Cr} / \mathrm{Au}$ contacts represent the pads of the resistor; (b) SEM image of a portion of the MLG-based device (enclosed in the dashed frame) after the Au-NPs' deposition. The clusters of Au-NPs are shown in the blue frames.

\section{Results and Discussion}

Scanning electron microscope (SEM) images (Figure 1b) show small clusters of Au-NPs. The NPs homogenously cover around 7\% of the device area, as determined from the SEM images. Pristine and functionalised devices show an Ohmic behaviour. Due to the sparse level of NP distribution, the graphene sheet resistance decreases only by $\sim 5 \%$ from $1 \mathrm{k} \Omega$ to $0.96 \mathrm{k} \Omega$.

Figure 2a reports the gas test before the NP deposition. $\mathrm{NO}_{2}$ is an electron-acceptor gas. The current increase indicates the p-doping of the pristine MLG. The signal keeps rising until the $\mathrm{NO}_{2}$ flow is stopped, demonstrating the absence of a steady-state in pristine MLG.

After the functionalisation, the chemical bonding between the MLG and Au-NPs causes n-type doping of the MLG, which results in a decrease in current upon $\mathrm{NO}_{2}$ exposure (Figure $2 \mathrm{~b}$ ). Interestingly the signal then demonstrates saturation. The $\Delta \mathrm{I} / \mathrm{I}_{0}$ is also positively affected by the NPs presence, indicating enhanced sensitivity. These results demonstrate that selectively deposited NP can dramatically change the gas sensing behaviour of graphene, enabling new ways to functionalise graphene-based gas sensors.

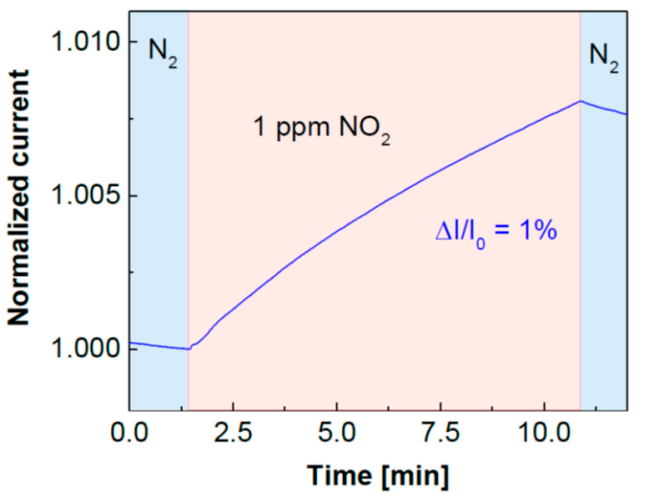

(a)

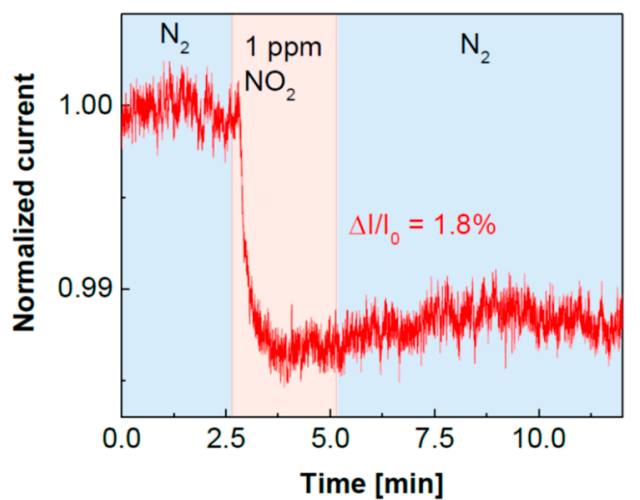

(b)

Figure 2. Dynamic current behaviour of the sensors, where the current is normalised to the value at the gas inlet. (a) pristine sensor; (b) sensor functionalised with Au-NP. 


\section{References}

1. Eriksson, J.; Puglisi, D.; Hsuan, Y.; Rositza, K.; Anita, Y.; Spetz, L. Adjusting the electronic properties and gas reactivity of epitaxial graphene by thin surface metallization. Phys. B Condens. Matter 2014, 439, 105108.

2. Wu, Y.; Jiang, W.; Ren, Y.; Cai, W.; Lee, W.H.; Li, H.; Piner, R.D.; Pope, C.W.; Hao, Y.; Ji, H.; et al. Tuning the Doping Type and Level of Graphene with Different Gold Configurations. Small 2012, 8, 3129-3136.

3. Vollebregt, S.; Alfano, B.; Ricciardella, F.; Giesbers, A.J.M.; Grachova, Y.; van Zeijl, H.W.; Polichetti, T.; Sarro, P.M. A transfer-free wafer-scale CVD graphene fabrication process for MEMS/NEMS sensors. In Proceedings of the 29th IEEE International Conference of Micro Electro Mechanical Systems, Shanghai, China, 24-28 January 2016; pp. 17-20.

4. Ricciardella, F.; Vollebregt, S.; Polichetti, T.; Alfano, B.; Massera, E.; Sarro, P.M. High sensitive gas sensors realized by a transfer-free process of CVD graphene. In Proceedings of the IEEE Sensors, Orlando, FL, USA, 31 October 2016; pp. 697-699.

Publisher's Note: MDPI stays neutral with regard to jurisdictional claims in published maps and institutional affiliations.

(C) 2020 by the authors. Licensee MDPI, Basel, Switzerland. This article is an open access article distributed under the terms and conditions of the Creative Commons Attribution (CC BY) license (http://creativecommons.org/licenses/by/4.0/). 